\title{
ON THE STABILITY ANALYSIS OF WEIGHTED AVERAGE FINITE DIFFERENCE METHODS FOR FRACTIONAL WAVE EQUATION
}

\section{N. H. Sweilam, M. M. Khader And M. Adel}

Abstract. In this article, a numerical study for the fractional wave equations is introduced by using a class of finite difference methods. These methods are extension of the weighted average methods for ordinary (non-fractional) wave equations. The stability analysis of the proposed methods is given by a recently proposed procedure similar to the standard John von Neumann stability analysis. Simple and accurate stability criterion valid for different discretization schemes of the fractional derivative, arbitrary weight factor, and arbitrary order of the fractional derivative, is given and checked numerically. Numerical test example and comparisons have been presented for clarity.

Mathematics subject classification (2010): 35L05, 26A33.

Keywords and phrases: Weighted average finite difference approximations; Fractional order wave equation; Stability analysis.

\section{REFERENCES}

[1] R. L. Bagley, and R. A. Calico, Fractional-order state equations for the control of viscoelastic damped structures, J. Guidance Control Dynamics, 14, 2 (1999), 304-311.

[2] D. A. Benson, S. W. Wheatcraft, and M. M. Meerschaert, Water Resour. Res. 36, 6 (2000), 1403-1412.

[3] A. V. Chechkin, V. Y. Gonchar, J. Klafter, R. Metzler, and L. V. Tanatarov, Lévy flights in a steep potential well, J. Stat. Phys., 115, (2004), 1505-1535.

[4] V. Daftardar Gejji, and H. Jafari, Solving a multi-order fractional differential equation using Adomian decomposition method, Appl. Math. Comput., 189, 1 (2007), 541-548.

[5] K. Diethelm, and N. J. Ford, Multi-order fractional differential equations and their numerical solution, Appl. Math. Comput., 154, (2004), 621-640.

[6] R. Hilfer, Applications of Fractional Calculus in Physics, World Scientific, Singapore, (2000).

[7] M. Ichise, Y. Nagayanagi, and T. Kojima, J. Electroanal. Chem. Interfacial Electrochem, 33, (1971), 253-265.

[8] M. M. Khader, On the numerical solutions for the fractional diffusion equation, Communications in Nonlinear Science and Numerical Simulations, 16, (2011), 2535-2542.

[9] A. A. Kilbas, H. M. Srivastava and J. J. Trujillo, Theory and Applications of Fractional Differential Equations, Elsevier, San Diego, (2006).

[10] F. Liu, V. Anh, I. Turner, and P. Zhuang, Time fractional advection-dispersion equation, Appl. Math. Comput., (2003), 233-246.

[11] C. Lubich, Discretized fractional calculus, SIAM J. Math. Anal., 17, (1986), 704-719.

[12] R. J. Mark, and M. W. Hall, Differintegral interpolation from a bandlimited signal's samples, IEEE Trans. Acoust. Speech Signal Process, 29, 6 (1981), 872-877.

[13] R. Metzler, and J. Klafter, Boundary value problems for fractional diffusion equations, Physica A, 278, (2000), 107-125. 
[14] K. S. Miller, and B. Ross, An Introduction to the Fractional Calculus and Fractional Differential Equations, John Wiley and Sons, (1993).

[15] K. W. Morton, and D. F. Mayers, Numerical Solution of Partial Differential Equations, Cambridge University Press, Cambridge, (1994).

[16] K. B. Oldham, and J. Spanier, Fractional Calculus: Theory and Applications, Differentiation and Integration to Arbitrary Order, Academic Press, New York, (1974).

[17] I. Podlubny, Fractional Differential Equations, Academic Press, San Diego, (1999).

[18] W. H. Press, S. A. Teukolsky, W. T. Vetterling, and B. P. Flannery, The Art of Scientific Computing, second ed., Cambridge University Press, Cambridge, (1992).

[19] I. M. Sokolov, J. Klafter, and A. Blumen, Fractional kinetics, Phys. Today, 55, (2002), 48-54.

[20] N. H. Sweilam, M. M. Khader and R. F. Al-Bar, Numerical studies for a multi-order fractional differential equation, Physics Letters A, 371, (2007), 26-33.

[21] N. H. Sweilam, M. M. Khader and A. M. Nagy, Numerical solution of two-sided space-fractional wave equation using finite difference method, Journal of Computational and Applied Mathematics, 235, (2011), 2832-2841.

[22] N. H. Sweilam, M. M. Khader and A. M. S. Mahdy, Crank-Nicolson finite difference method for solving time-fractional diffusion equation, Journal of Fractional Calculus and Applications, 2, 2 (2012), $1-9$.

[23] S. B. Yuste, and L. Acedo, An explicit finite difference method and a new von Neumann-type stability analysis for fractional diffusion equations, SIAM J. Numer. Anal., 42, (2005), 1862-1874.

[24] S. B. Yuste, Weighted average finite difference methods for fractional diffusion equations, Journal of Computational Physics, 216, (2006), 264-274. 